\title{
Instituto Multidisciplinar de Materiais Poliméricos
}

O Ministério da Ciência e Tecnologia (MCT) criou em 2001 um novo programa de fomento à pesquisa e desenvolvimento, em parceria com o Banco Mundial. Trata-se do Programa Institutos do Milênio, que visa a promover iniciativas multidisciplinares e multiinstitucionais, na forma de redes de pesquisa, dentro da política do MCT de contemplar demandas da cadeia produtiva e o fortalecimento das ciências básicas. Mais de 200 projetos foram submetidos para apreciação do MCT, dos quais 17 foram selecionados por uma equipe de avaliadores do Brasil e do exterior. Cada instituto deverá receber recursos da ordem de alguns milhões de reais por um periodo de 3 anos. Um dos Institutos do Milênio escolhidos é o Instituto Multidisciplinar de Materiais Poliméricos (IMMP), coordenado pelo Prof. Roberto Mendonça Faria, do Instituto de Física de São Carlos, USP. O IMMP é formado por cerca de 140 pesquisadores, sendo mais de 60 doutores, de 17 instituições das 5 regiões do país (vide lista no final da matéria). Participam do IMMP especialistas em física, química e engenharia de polímeros, além de profissionais dedicados à transferência de tecnologia.

Um Workshop em Atibaia, de 24 a 27 de março de 2002, marcou o início das atividades do IMMP. No evento foram estabelecidos metas e projetos articulados, cada um sob responsabilidade direta de um pesquisador "líder-de-projeto", mas com tarefas distribuídas entre todos os grupos que tenham a competência específica para o dado projeto. $\mathrm{O}$ ponto comum que une os componentes do IMMP é o estudo de propriedades físicas e químicas de diversos materiais poliméricos, dos pontos de vista teórico e experimental, e suas aplicações. Serão investigados polímeros isolantes, polímeros ferroelétricos, polímeros eletrônicos, polímeros fotônicos e biopolímeros.

Para os polímeros isolantes, em particular, o estudo de propriedades elétricas deverá subsidiar a identificação de materiais poliméricos e condições que minimizem as rupturas elétricas e estendam o tempo de vida de isoladores elétricos. O impacto esperado aqui é principalmente econômico, com o desenvolvimento de produtos e processos que possam ser transferidos para as indústrias de tecnologia e empresas concessionárias de energia. Neste contexto, será retomada no IMMP uma parceria que realizou projetos no Instituto de Energia e Eletrotécnica da USP (projetos MATRs financiados pela CESP, ELETROPAULO e CPFL), que reverteram em significativa redução nas perdas de energia no sistema de distribuição. Para tanto, o IMMP conta com a participação do Lactec do Paraná, que já acumula experiência nesta área e tem caráter de transferidor de tecnologia para as Concessionárias de Energia e Empresas fornecedoras de cabos e acessórios.

Uma outra preocupação do IMMP é com a atual dependência do Brasil em dispositivos elétricos e eletrônicos em geral, que tem forte influência negativa na balança comercial. Por isso, esforços serão feitos no IMMP para que políme- ros sejam aplicados em optoeletrônica e fotônica, permitindo uma importante recuperação econômica no setor, com benefícios sociais através da geração de empregos e elevação do padrão tecnológico da Nação. Dentre as várias possíveis aplicações merece destaque a fabricação de transistores de efeito de campo (FETs), diodos eletroluminescentes (LEDs), diodos fotovoltaicos e dispositivos fotocondutores, obtidos de polímeros condutores, na qual integrantes do IMMP já vêm trabalhando. Abaixo é apresentado o esquema básico de um LED polimérico e um esquema de um display de matriz passiva.

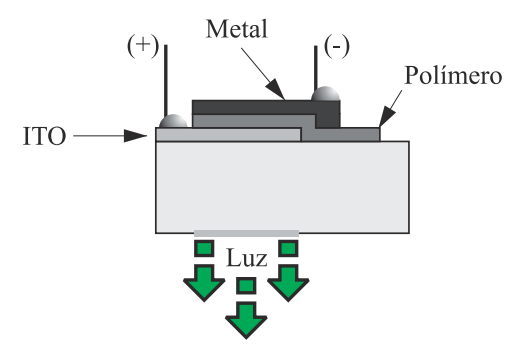

Estrutura de um LED 


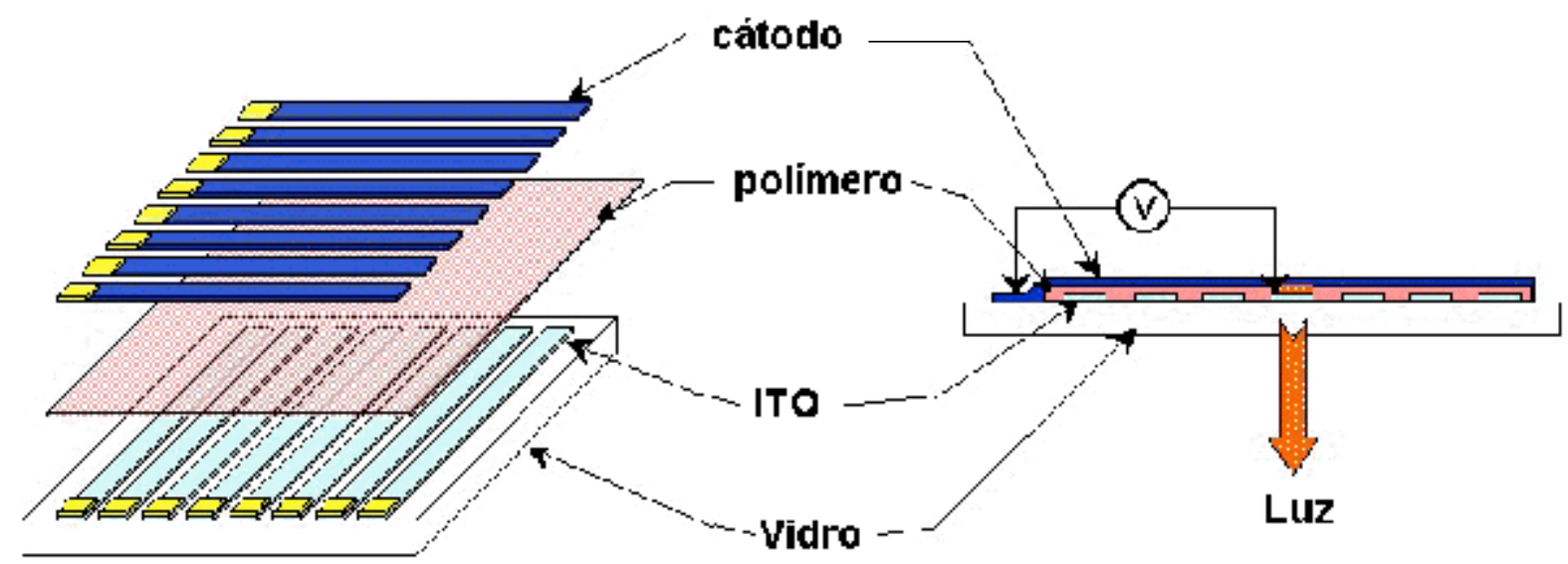

Esquema de um display de matriz passiva

Os processos de fabricação de dispositivos eletrônicos com polímeros são simples e podem ser feitos com investimentos relativamente modestos. A tecnologia de dispositivos com materiais poliméricos está ainda em sua infância sendo este o momento propício "para entrar", visto que o Brasil hoje possui uma capacitação de nível internacional na área. Estes dispositivos representarão um mercado estratégico de enorme dimensão, com projeção de chegar a US\$ 3 bilhões no ano 2005 só para "displays" e LEDs sinalizadores.

Outras aplicações dos polímeros eletrônicos incluem conversão de energia fotovoltaica, em que polímeros conjugados começam a dar resultados de eficiência bastante animadores; sensores de gases, de pressão e de radiação infravermelha e de cápsulas eletroacústicas. Esses materiais terão, também, aplicações em medicina (pela compatibilidade dos polímeros com os tecidos vivos) e de instrumentação médica. Num futuro breve, pode-se também vislumbrar a utilização de polímeros luminescentes em iluminação de ambientes, o que certamente trará uma revolução no setor. Para os polímeros fotônicos, a exploração de novos materiais para dispositivos ópticos, de baixo custo, pode gerar sensíveis melhoras no sistema de comunicação óptica. Os polímeros ferroelétricos podem ser empregados em dispositivos eletroacústicos e piroelétricos. A biocompatibilidade de biopolímeros também pode ser explorada em diversas aplicações.

A transformação de resultados de pesquisa em tecnologia é obviamente um grande desafio. No IMMP, além da já mencionada participação do Lactec, participará também o Genius Instituto de Tecnologia, sediado em Manaus, que atua em transferência de tecnologia.

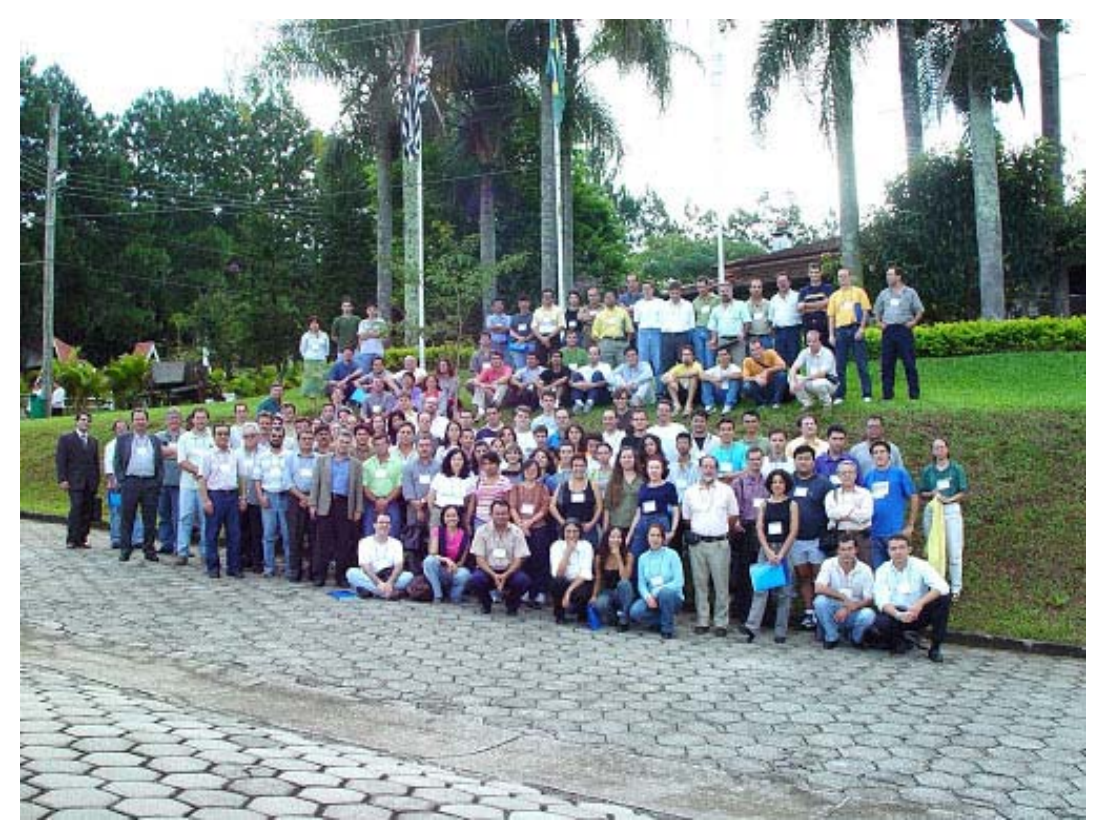

Presentes no workshop ocorrido em Atibaia
Para o desenvolvimento de das, será necessário um grande esforço de pesquisa fundamental, quer seja na síntese e caracterização de novos materiais poliméricos, ou no estudo das propriedades elétricas, ópticas e de processamento desses materiais. Ressalte-se que alguns dos materiais ou tópicos sob investigação estão na fronteira do conhecimento de novos materiais e nanotecnologia. Para tanto o IMMP reúne especialistas, experimentais e teóricos, das diversas áreas envolvidas, e que estarão qualquer das aplicações menciona- 
trabalhando em cooperação na rede do IMMP e também com pesquisadores de outras instituições no Brasil e no exterior. A título de ilustração, um dos colaboradores do IMMP é o Prof. Alan MacDiarmid, da Universidade da Pensilvânia, EUA, ganhador do Prêmio Nobel de Química de 2000. Juntamente com A. Heeger e H. Shirakawa, o Prof. MacDiarmid foi agraciado com o Nobel não só pela inovação nos métodos de síntese e desenvolvimento dos modelos teóricos que explicaram o caráter semicondutor dos polímeros sintéticos, mas tam- bém pela demonstração inequívoca do seu potencial tecnológico. Isso ilustra a necessidade de uma ação concentrada em pesquisa fundamental e na busca por aplicações e transferência de tecnologia, como é a filosofia do IMMP.

\title{
Instituições envolvidas
}

Departamento de Engenharia de Materiais da UFSCar

Departamento de Engenharia Química da UFRN

Departamento de Física da UFPI

Departamento de Química da UFPR

Escola Politécnica da USP

Fac. de Ciências e Tecnologia de Presidente Prudente da UNESP

Fac. de Engenharia de llha Solteira da UNESP

Fac. de Filosofia Ciências e Letras de Ribeirão Preto da USP

Genius Instituto de Tecnologia
Instituto de Ciências Exatas e da Terra da UFMT

Instituto de Física da UNICAMP

Instituto de Física da USP

Instituto de Física de São Carlos da USP

Instituto de Geociências e Ciências Exatas de

Rio Claro da UNESP

Instituto de Química da UNICAMP

Instituto de Química da USP

LACTEC Instituto de Tecnologia para o Desenvolvimento

Matéria preparada pelos Profs. Osvaldo N. Oliveira Jr. e Roberto M. Faria, Instituto de Física de São Carlos, USP

\section{SÓCIOS PATROCINADORES DA ABPOI}

\author{
Basf S/A \\ Ciba Especialidades Químicas Ltda. \\ DSM South America Ltda. \\ GE Plastics South America S/A \\ OPP Química S/A \\ Polibrasil Resinas S/A \\ Rhodia Poliamida Ltda.
}


GE Plastics 